\section{Cervical cancer}

\section{INNOVATV 301/ENGOT-CX12/GOG-3057: TISOTUMAB VEDOTIN VS INVESTIGATOR'S CHOICE CHEMO IN SECOND- OR THIRD-LINE RECURRENT OR METASTATIC CERVICAL CANCER}

${ }^{1}$ IB Vergote*, ${ }^{2} \mathrm{~L}$ Randall, ${ }^{3} \mathrm{E}$ Kalbacher, ${ }^{4} \mathrm{~K}$ Madsen, ${ }^{1} \mathrm{E}$ Van Nieuwenhuysen, ${ }^{5} \mathrm{~A}$ GonzalezMartin, ${ }^{6} \mathrm{D}$ Lorusso, ${ }^{7}$ I Soumaoro, ${ }^{8} \mathrm{~S}$ Jain, ${ }^{9} \mathrm{~B}$ Slomovitz. ${ }^{1}$ Belgium and Luxembourg Gynaecological Oncology Group, University of Leuven, Leuven Cancer Institute, Leuven, Belgium; ${ }^{2}$ Virginia Commonwealth University, Massey Cancer Center, Richmond, VA, USA; ${ }^{3}$ Groupe d'Investigateurs Nationaux pour l'Etude des Cancers Ovariens, CHRU Jean Minjoz, Besançon, France; ${ }^{4}$ Rigshospitalet, University Hospital of Copenhagen, Nordic Society of Gynaecological Oncology Clinical Trial Unit (NSGO-CTU), Centre for Cancer and Organ Diseases, Copenhagen, Denmark; ${ }^{5}$ Grupo Español de Investigación en Cáncer de Ovario (GEICO), Clinica Universidad de Navarra, Department of Medical Oncology, Madrid, Spain; ${ }^{6}$ Multicentre Italian Trials in Ovarian Cancer and Gynaecological Malignancies Group (MITO) and Scientific Directorate and Department of Women and Child Health, Fondazione Policlinico Universitario Agostino Gemelli IRCCS, Rome, Italy; 'Genmab US, Inc., Plainsboro, NJ, USA; ${ }^{8}$ Seagen Inc., Bothell, WA, USA; ${ }^{9}$ Broward Health, Fort Lauderdale, FL, USA

\subsection{6/ijgc-2021-ESGO.1}

Introduction/Background* Doublet chemotherapy (paclitaxel plus either platinum or topotecan) with bevacizumab (if eligible) is recommended for first-line treatment of recurrent or metastatic cervical cancer ( $\mathrm{r} / \mathrm{mCC}$; Tewari 2014). In the second-line setting, there are limited data for available treatment options.

Tisotumab vedotin (TV) is an investigational antibody-drug conjugate directed to tissue factor. In the phase 2 pivotal trial (innovaTV 204/ENGOT-cx6/GOG-3023) in $\mathrm{r} / \mathrm{mCC}$ patients with disease progression on or after chemotherapy, TV demonstrated clinically meaningful and durable activity (objective response rate [ORR]: 24\%; median duration of response [DOR]: 8.3 months) with a manageable and tolerable safety profile. Most adverse events associated with TV were mild to moderate. These findings support further investigation of TV in patients with $\mathrm{r} / \mathrm{mCC}$ who progress on first-line treatment options.

Methodology innovaTV 301/ENGOT-cx12/GOG-3057 (NCT04697628) is a global, randomized, open-label, phase 3 trial evaluating efficacy and safety of TV in patients with previously treated $\mathrm{r} / \mathrm{mCC}$. Eligible patients must be $\geq 18$ years, have $\mathrm{r} / \mathrm{mCC}$, and have progressed after receiving 1-2 prior lines of therapy (either standard of care systemic chemotherapy doublet or platinum-based therapy with bevacizumab, if eligible).

Approximately 482 patients will be randomized $1: 1$ to receive 21-day cycles of TV $(2.0 \mathrm{mg} / \mathrm{kg}$ IV once every 3 weeks) or investigator's choice of chemotherapy: topotecan, vinorelbine, gemcitabine, irinotecan, or pemetrexed. The primary endpoint is overall survival. Key secondary endpoints are progression-free survival, ORR, time to response, DOR, safety, and quality of life outcomes. The study is enrolling and will have sites in the USA, Europe, Japan, Latin America, Taiwan, Singapore, and South Korea.

Result(s)* Not applicable for trial in progress

Conclusion* Not applicable for trial in progress

\section{ALPELISIB FOR RECURRENT PIK3CA-MUTATED} RECURRENT CERVCIAL CANCER

${ }^{1} \mathrm{G}$ Bogani*, ${ }^{2} \mathrm{~A}$ Indini, ${ }^{1} \mathrm{M}$ Bini, ${ }^{1} \mathrm{~F}$ Raspagliesi*. ${ }^{1}$ Fondazione IRCCS Istituto Nazionale dei Tumori, Milano, Italy; ${ }^{2}$ Policlinico di Milano, Medical Oncology, Milan, Italy

10.1136/ijgc-2021-ESGO.2
Introduction/Background* Advanced/recurrent cervical cancer has limited therapeutic options, with a median progressionfree survival (PFS) after the failure of systemic treatments ranging between 3.5 and 4.5 months. Here, we reported our preliminary experience in the use of BYL719 (alpelisib) in advanced/recurrent cervical cancer after failure of at least 2 lines of treatment.

Methodology The Fondazione IRCCS Istituto Nazionale dei Tumori di Milano (Italy) approved this prospective investigation. From 04/01/2020 to 09/01/2020, 17 consecutive patients with recurrent cervical cancer underwent next generation sequencing (NGS) to assess the presence of PIK3CA mutation/ alteration.

Result(s)* Overall, 17 patients were tested for PIK3CA mutation/alteration. PIK3CA mutation was detected in seven (41\%) patients. Six patients were included in the study; one patient was diagnosed with a synchronous tumor during the screening phase. All patients had been treated with at least 2 previous lines of systemic treatment: 3 patients received $>2$ prior lines of treatment in the recurrent or metastatic setting; 60\% had received prior bevacizumab in combination with chemotherapy. All patients started alpelisib at the daily dosage of $300 \mathrm{mg}$. Investigator-assessed confirmed objective response rate (ORR) was 33\%. The disease control rate (DCR) was 100\%. According to the RECIST 1.1, two patients had a partial response (PR), and four patients had stable disease (SD). No complete response was observed. The mean duration of response (DOR) was 6.6 (SD 3.75) months; four patients had PR lasting for $>6$ months. One patient stopped the treatment at 0.82 months due to the onset of a grade 2 adverse event (AE) (skin rash). Grade 3 treatment-related AEs included: lymphoedema $(n=1,20 \%)$ and rash $(n=1,20 \%)$. No treatment-related grade 4-5 AEs occurred.

Conclusion* Further trials are needed to assess the safety and effectiveness of alpelisib in PIK3CA-mutated recurrent/ advanced cervical cancer

\section{RADICAL TRACHELECTOMY. EXPERIENCE IN KAIOR}

${ }^{1}$ A Satanova*, ${ }^{2} \mathrm{D}$ Kaidarova, ${ }^{1} \mathrm{Y}$ Kukubassov, ${ }^{3} \mathrm{R}$ Bolatbekova, ${ }^{1} \mathrm{D}$ Kaldybekov. ${ }^{1}$ Kazakh Institute of Oncology and Radiology, Oncogynecology, Almaty, Kazakhstan; ${ }^{2}$ Kazakh Institute of Oncology and Radiology, Head of Institute, Almaty, Kazakhstan; ${ }^{3}$ Almaty Oncological Center, Oncogynecology, Almaty, Kazakhstan

\subsection{6/ijgc-2021-ESG0.3}

Introduction/Background* Radical trachelectomy with pelvic lymphadenectomy, originally described by Dargent et al. in 1988 became a new treatment option for young patients in the world with early invasive cervical cancer who want to maintain their fertility. Recent data indicate that cancer outcomes after radiation therapy are comparable to those after standard radical hysterectomy .Fertility-sparing radical trachelectomy was revolutionary when it first appeared. This procedure now allows patients to survive cancer and save uterus for future bearing of the child. Initially, they approached him as a vaginal procedure with laparoscopic evaluation. This approach is comparable to type B or modified radical hysterectomy, but with greater limited resection of parametrium.

Methodology statistical analysis

Result(s)* Since 2013, radical trachelectomy has been performed at KazIOR. From 2013 to 2021, 8 operations were performed, 7 of them by abdominal access, 3 by laparoscopic approach. $6(75 \%)$ of the patients had stage 1 in 1 from 2 to 
$4 \mathrm{~cm} ; 2$ (25\%) had a 1a1 stage. No patient had a tumor larger than $4 \mathrm{~cm}$ on examination, MRI, and histological examination. No patient received neoadjuvant chemotherapy. 2 patients (25\%) underwent a biopsy for the purpose of establishing a diagnosis, 6 patients (75\%) conization of the cervix. The average age of the patients was 28 years (from 26 to 37 years). $5(62.5 \%)$ patients were nulliparous, 2 patients had 2 children, 1 patient had 1 child.

LVSI was negative in preoperative histological examination, and resection margins were also negative. In 1 patient $(12.5 \%)$ after histological examination LVSI was positive, in 7 it was negative. Of the 8 patients who retained fertility, there were 5 pregnancies, 2 miscarriages at 9-10 weeks, and 3 deliveries at 36-37 weeks of gestation.

Conclusion* Thus, in 2013-2021, 8 radical trachelectomy operations were successfully performed. The data presented in this publication demonstrate that patients with stage IB1 tumors ranging in size from 2 to $4 \mathrm{~cm}$ and with favorable histology are acceptable candidates for attempted radical trachelectomy. These patients must be clearly informed of all the options if they want to consider maintaining fertility.

\section{SINGLE INSTITUTION OUTCOMES FOR CERVICAL CANCER PATIENTS TREATED WITH PALLIATIVE SYSTEMIC ANTI CANCER THERAPY}

${ }^{1} \mathrm{C}$ Cotronei ${ }^{*},{ }^{2} \mathrm{~A}$ Stillie, ${ }^{2} \mathrm{M}$ Zahra. ${ }^{1}$ University of Edinburgh, UK; ${ }^{2}$ Western General Hospital Edinburgh Cancer Centre, Edinburgh, UK

10.1136/ijgc-2021-ESGO.4

Aim Bevacizumab, in combination with chemotherapy, has been implemented as first line treatment of patients with metastatic cervical cancer since 2015. This is a single institution review to evaluate the overall survival (OS) and progression free survival (PFS) for patients with advanced cervical cancer treated with palliative systemic anti cancer therapy (SACT) over a 5-year period from 2015-2020.

Methodology Patient records were reviewed retrospectively for all cervical cancer patients who received palliative SACT in a single institution between 2015-2020. Patient demographics, previous treatment for cervical cancer (if applicable), palliative SACT regimen received, toxicities, and outcome data were recorded. OS and PFS were calculated for the whole cohort and for the following subgroups: patients treated with Bevacizumab in combination with palliative chemotherapy; patients treated with chemotherapy alone. Toxicities were recorded by Common Toxicity Criteria (CTC).

Result(s)* 52 patients received palliative SACT during the 5year period following a diagnosis of metastatic cervical cancer. The median age was 51 (range 25-76). The majority of patients had a squamous cell histology (75\%). 40 patients received Bevacizumab in combination with chemotherapy, 12 were treated with chemotherapy alone due to comorbidities. The median OS for the whole patient cohort was 64 weeks and PFS was 45 weeks. Patients who received bevacizumab in combination with chemotherapy had a median OS of 76 weeks compared to 50 weeks in the chemotherapy only subgroup. Grade 1 and 2 toxicites were comparable in patients receiving chemotherapy alone and those treated with Bevacizumab. $10 \%$ of patients in the cohort receiving bevacizumab developed a thromboembolic event compared with 5.5\% in the chemotherapy only cohort.
Conclusion* Results from this study of real world data are comparable with the published data. The results support the continued use of Bevacizumab, in combination with chemotherapy, in our patient population as the combination regimen improves OS with acceptable toxicties.

\section{COMPARISON OF LOCALLY ADVANCED CERVICAL CANCER TREATMENT GUIDELINES IN EUROPE}

${ }^{1} \mathrm{~A}$ Leary*, ${ }^{2} \mathrm{~B}$ Monk, ${ }^{3} \mathrm{~J}$ Takyar, ${ }^{4} \mathrm{~A}$ Nunes, ${ }^{4} \mathrm{JD}$ Hernández Chagüi, ${ }^{4} \mathrm{~K}$ Rabon-Stith, ${ }^{5}$ E Pujade-Lauraine. 'Gustave Roussy Cancer Center, University Paris Saclay, Department of Medical Oncology, Villejuif, France; ' ${ }^{2}$ University of Arizona College of Medicine, Creighton University School of Medicine, Division of Gynecologic Oncology, Phoenix, USA; ${ }^{3}$ Parexel International, HEOR Evidence Evaluation Regulatory and Access, Mohali, India; ${ }^{4}$ AstraZeneca, Gaithersburg, USA; ${ }^{5}$ Arcagy-Gineco, Paris, USA

\subsection{6/ijgc-2021-ESG0.5}

Introduction/Background* From our previously conducted global systematic literature review (SLR) assessing recommended treatment for locally advanced cervical cancer (LACC) it was observed that there was international consensus on the use of concurrent chemoradiotherapy (cCRT) as standard of care (SoC) for Stage IIB-IVA LACC, but recommendations for Stage IB2-IIA LACC varied. Here, we present a subanalysis of European guidelines to determine if recommended LACC treatment is consistent across Europe.

Methodology English-language cervical cancer treatment guidelines and consensus statements were identified through literature databases (1999-2020), international and national health organizations, and general internet searches. Included guidelines represented the latest updates from Europe. Non-English guidelines from EU-5 countries were translated for comparison (Italian [AIOM], German [S3], and French [HAS]) to the 4 European guidelines obtained through this SLR protocol (ESTRO/ESGO/ESP, ESMO, Spain [SEOM], UK [NHS]). An English-language guideline from Germany was excluded because a newer German-language only version was available. Result(s)* The 7 guidelines were updated between 2010 and 2021 (table 1). ESTRO/ESGO/ESP guidelines used AJCC 8th edition TNM criteria for staging. All others, except SEOM, used FIGO 2009 criteria; SEOM used FIGO 2018 criteria. Table 1 shows recommended treatment by LACC stage. For Stage IB2-IIA2 disease, cCRT was the SoC or an alternative to radical hysterectomy. AIOM was the only guideline to recommend radiotherapy alone for Stage IIA1 disease. cCRT followed by brachytherapy was SoC for suitable patients with Stage IIB-IVA LACC among all European guidelines. For cCRT, a platinum-based regimen was recommended by all, and most guidelines recommended an external beam radiation therapy (EBRT) dose of 45-50 Gy (table 2).

Conclusion* In line with global SLR findings, consensus on the use of CCRT as primary treatment for Stage IIB-IVA LACC was recommended by European guidelines. The ideal cCRT regimen was weekly cisplatin at $40 \mathrm{mg} / \mathrm{m} 2$ concurrent with EBRT at 45-50 Gy and followed by brachytherapy. For Stage IB-IIA LACC, recommendations mainly varied between radical hysterectomy and cCRT as options or cCRT alone. Alignment of guidelines with FIGO 2018 staging criteria may help reduce variation in recommended treatment for earlystage LACC.

Funding AstraZeneca

Klikně te nebo klepně te sem a zadejte text. 